\title{
ERRATUM: The cross sections of fusion- evaporation reactions: the most promising route to superheavy elements beyond $Z=118$
}

\author{
Khuyagbaatar Jadambaa ${ }^{1,2}$ \\ ${ }^{1}$ Helmholtz Institute Mainz, 55099 Mainz, Germany \\ ${ }^{2}$ GSI Helmholtzzentrum für Schwerionenforschung, 64291 Darmstadt, Germany \\ Original article: \\ EPJ Web of Conferences 163, 00030 (2017), DOI: 10.1051/epjconf/201716300030
}

Figure 1 on page 2 should be replaced by the following, corrected version. The caption remains unchanged.

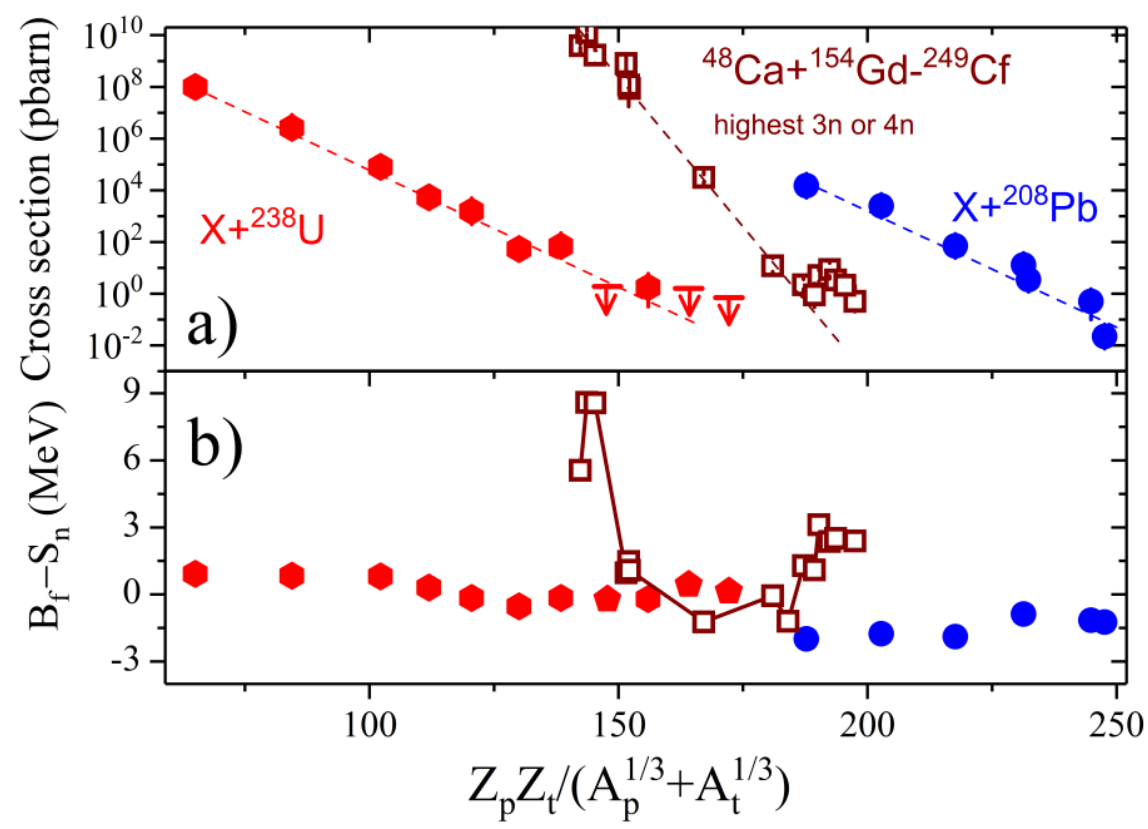

Figure 1. a) Compiled experimental ER cross sections of fusion-evaporation reactions with ${ }^{208} \mathrm{~Pb}$ and ${ }^{238} \mathrm{U}$ targets, and ${ }^{48} \mathrm{Ca}$ projectiles [28-32]. Only maximum values of $\sigma_{1 \mathrm{n}}$ and $\sigma_{5 \mathrm{n}}$ are shown for ${ }^{208} \mathrm{~Pb}$ and ${ }^{238} \mathrm{U}$, respectively. Maximum values of either $\sigma_{3 \mathrm{n}}$ or $\sigma_{4 \mathrm{n}}$ from ${ }^{48} \mathrm{Ca}$-induced reactions with deformed Gd-Cf targets are shown. Arrows mark upper limits. Dashed lines are drawn to guide an exponential descent. b) Theoretical $\mathrm{B}_{\mathrm{f}}-\mathrm{S}_{\mathrm{n}}$ calculated within the macro-microscopic FRDM approach are given $[3,37]$. 